\title{
Stop Making Sense of Bell's Theorem and Nonlocality?
}

\author{
Federico Laudisa \\ Department of Human Sciences, University of Milan-Bicocca \\ Piazza dell'Ateneo Nuovo 1, 20126 Milan, Italy
}

\begin{abstract}
In a recent paper on Foundations of Physics, Stephen Boughn reinforces a view that is more shared in the area of the foundations of quantum mechanics than it would deserve, a view according to which quantum mechanics does not require nonlocality of any kind and the common interpretation of Bell theorem as a nonlocality result is based on a misunderstanding. In the present paper I argue that this view is based on an incorrect reading of the presuppositions of the EPR argument and the Bell theorem and, as a consequence, is unfounded.
\end{abstract}

\section{Introduction}

Since the original publication in 1935, the EPR paper does not stop to puzzle physicists and philosophers alike. Even worse for the Bell nonlocality theorem: should the natural world be nonlocal at a certain scale - as many believe - this is increasingly thought to be a possible sign that our beloved theories of space and time might be far from fundamental. We know that quantum mechanics allows for certain physical systems that have interacted to retain a sort of mutual influence across large distances - 'large' in a sense in which the systems should be totally disconnected and independent in a relativistic spacetime framework - although such 'influence' turns out not to be strong enough to produce a real communication or physically significant exchange at-a-distance. According to a serious reading of Bell's result, if the above can be summarized by the claim that quantum theory is a non-local but non-signalling theory, Bell's theorem establishes that this status is not an accidental feature of a single theory, but applies to any theory that is supposed to extend quantum mechanics by incorporating its 
statistical predictions. Given the effort that appears to be necessary in order to take into account the implications of this theorem for our conceptions of physical reality and its embedding into spacetime, some resistance to such a straightforward reading of the Bell result is reasonable: nevertheless, even those who endorse such 'resisting' option are not exempt from the obligation of a correct formulation of the state of the art.

In this vein, the attempts to deflate the revolutionary impact of the theorem started very early and, with different tools and aims, still continue today. The most common of these attempts has been the reading of the Bell theorem as a no-hidden-variables result, a reading that is blatantly contradicted by the very existence of a consistent and well-developed 'hidden-variables' formulation of quantum mechanics such as the De Broglie-Bohm formulation or Bohmian mechanics ${ }^{1}$. The more recent version of this deflationary attitude tries to refine the no-hidden-variables issue in terms of a vague 'realism', a (far from well specified) condition whose conjunction with locality would be the alleged target of the theorem. Thus what is supposed to be the focus of the latter, jointly with the other (obvious) assumption that quantum-mechanical predictions are to preserved, is summarized in the expression local realism, an instance of which can be the following:

Quantum theory predicts correlations between spacelike separated events, which are nonsignaling but cannot be explained within local realism, i.e., within the framework in which all outcomes have preexisting values for any possible measurement before the measurements are made («realism») and where these values are independent from any action at spacelike separated regions («locality»). (Pawlowski, Brukner 2009, p. 030403-2)

The 'realistic' part of the local realism condition is often formulated, even recently, as the idea that physical systems are endowed with pre-existing properties that turn out to be independent of any measurement. Under the assumption of local realism, therefore, and provided quantum mechanics' predictions are taken for granted, a die-hard view takes the Bell theorem to be a result that does not establish non-locality but rather the impossibility of any objective (i.e. observer-independent in principle) account of the quantum phenomena. ${ }^{2}$

The claim that what the Bell theorem is about is the refutation of local realism has surprisingly acquired the status of a commonplace, to such an extent that any new

\footnotetext{
${ }^{1}$ For a recent review see Goldstein 2017. As is well-known, Bell himself disliked the 'hidden-variables' jargon and (rightly) considered the hidden-variables terminology seriously misleading. Moreover, needless to say, Bohmian mechanics is a non-local theory, consistently with Bell's theorem.

2 For an instructive sample of quotations on the centrality of 'local realism' see Norsen 2007, pp. 312-314, and Laudisa 2008, pp. 1113-1115.
} 
experimental confirmation of the nonlocal behaviour of entangled states - also in contexts inspired by quantum technology which are not interested into the philosophical side of the issue $^{3}$ - is advertized as a blow to 'local realism'. The vast majority of the (more or less) recent literature that takes local realism to be the target of the Bell theorem, however, is far from clear on what a realism assumption is supposed to require. If all seem to agree that, in order for realism to hold, the physical systems under scrutiny must be endowed with preexisting properties, it is far from straightforward whether - and to what extent - such properties (i) depend essentially or not on the measurement interactions to which the systems themselves may be subject, (ii) determine or not all the outcomes of possible measurements that can be performed on the physical systems. The two points (i) and (ii) are reminiscent of the widespread terms 'Non-Contextuality' and 'Determinism', respectively. The main recent defenders of the anti-realism project, who in my opinion completely misunderstand the meaning of the Bell theorem (see for instance Zukovski, Brukner 2014), are quite clear in presupposing (i) in a strong sense, namely the pre-existing properties do not depend on the measurement interactions in that they are passively revealed by the measurements themselves. ${ }^{4}$ Far less clear is whether they would endorse (ii) ${ }^{5}$.

To sum up: on the background of the above mentioned attempt to resist the radical implication of Bell's nonlocality for our ordinary spacetime theories, the interpretation focusing on local realism would allow one to preserve locality, by 'discharging' the import of the theorem on the side of "realism". Given the anti-realistic folklore surrounding quantum mechanics anyway since the inception of the theory, this move comes at a relatively little price and contributes to downplay significantly the foundational relevance of Bell's theorem.

\footnotetext{
${ }^{3}$ A recent example out of many similar is the nonlocality results concerning the so-called hypergraph states, a family of multiparticle quantum states that generalizes the well-known concept of Greenberger-Horne-Zeilinger states and that turn out to be interesting resources for quantum metrology and measurement-based quantum computation. Gachechiladze, Budroni and Gühne 2016 show that correlations in hypergraph states can be used to derive various types of nonlocality proofs: the term 'realism' occurs in the title but it plays no role whatsoever in the result.

${ }^{4}$ A similar view seems to be presupposed by many presentations of the consistent histories approach to QM: on the point, see the 2011 exchange on the Americal Journal of Physics between Griffiths and Maudlin (Griffiths 2011, Maudlin 2011).

${ }^{5}$ Curiously enough, an interpretation which is light-years remote from the "local-realistic" one, namely Bohmian mechanics, assumes not only that physical systems have at least some pre-existing properties, but also che that such properties - jointly with (suitable properties of) the measurement context - determine the measurement outcomes. Clearly Bohmian mechanics does not collapse into a 'local-realistic' interpretation because it is nonlocal by construction and takes the properties of the physical system to strongly depend on measurement interactions: but a symptom of how confused is the 'local-realistic' interpretation is just the circumstance that those who endorse it wish to free quantum theory from 'realism' without realizing how close some of their assumptions may be to a thoroughly realistic interpretation!
} 
A recent review of the issue (Boughn 2017) reiterates the point and misrepresents once again the presuppositions and general meaning of Bell's theorem: the present paper is intended to show why. Most of the points I will make refer to problems that are far from new, and some of them date back to the very year in which the nonlocality result was published: however, the current appearance of claims that appear to forget some basic, well-established points, useful to locate the EPR-nonlocality issue in the logical space that it deserves, makes it essential to revive them.

My paper will touch, in the order, (i) the incorrect formulation of the meaning of the Bell theorem, its logical premises and their relation to the EPR argument; (ii) the related role of the so-called counterfactual definiteness condition in the EPR argument that is alleged to carry over in the derivation of the Bell theorem; (iii) the claim that a locality condition which in fact equivalent to what is known as outcome independence (OI) is not violated in QM and finally (iv) the plausibility of an ambiguous claim by W.H. Furry concerning the meaning of the original EPR argument, a claim that Boughn defends in support of the idea that entanglement is not peculiar to quantum mechanics.

\section{2. 'Classicality' and the Bell theorem}

The key point in Boughn's arguments concerns the formulation itself of the Bell theorem that he provides ${ }^{6}$. This is his wording:

Bell's theorem provides a general test, in the form of an inequality, that the results of an EPR type gedanken experiment must satisfy if it is describable by any classical theory, even one with hidden variables, so long as such a model is locally causal. Bell then proceeded to demonstrate how the predictions of quantum mechanics violate this inequality. His conclusion was that any hidden variable theory designed to reproduce the predictions of quantum mechanics must necessarily be nonlocal and allow superluminal interactions. (Boughn 2017, p. 2)

[...] Bell's demonstration that any classical, hidden variable treatment of the system demands acausal, superluminal signals provides sufficient grounds to summarily dismiss such classically based models. (Boughn 2017, p. 3, italics added)

It is quite clear then that, according to the Boughn presentation, the Bell theorem has among its assumption a condition of classicality: a (far from clearly defined) condition, that it is

\footnotetext{
${ }^{6}$ The story of this misunderstanding is long: see for instance the recollection in Laudisa 2008, pp. 1113-1116.
} 
reasonable to interpret intuitively as the requirement that any physical system under scrutiny is supposed to be endowed at any given time with a bunch of robust properties, each of which unambiguously characterized and holding independently from any measurement interaction with the system (pre-existing properties is a suitable term that has been sometimes used to denote these alleged properties). This appears to be the sense that the term classicality is supposed to convey, the sense of a representation of the world in which the physical theory does nothing but 'disclose' a state of affairs that simply is what it is, in total independence from the questions that we scientists decide to raise about it ${ }^{7}$. That this is the intended interpretation is clear from another passage where, with reference to the mathematically precise formulation of the Bell locality, Boughn states:

To be sure, in his 1964 paper, Bell did not conclude that quantum mechanics is nonlocal, only that a classical hidden variable model designed to reproduce the statistical predictions of quantum mechanics must necessarily be nonlocal. However, in a subsequent paper, he formalized "a notion of local causality" that was directly applicable to quantum mechanics and concluded that quantum mechanics itself is nonlocal. This analysis used a mathematical expression of locality similar to the above direct product of probabilities. The other concept necessary for the derivation of Bell's original inequality was a notion of "classical realism", which in the 1964 paper takes the form of classical hidden variables and the counterfactual definiteness they imply. (Boughn 2017, p. 11, italics added)

In this vein, a passage from the Bell 1964 paper is invoked, a passage that verbatim seems to make the above 'classicality-inspired' readings admissible. It is the final statement of the paper, where Bell summarizes in a sketchy way the result proved in the preceding pages: "In a theory in which parameters are added to quantum mechanics to determine the results of individual measurements, without changing the statistical predictions, there must be a mechanism whereby the setting of one measurement device can influence the reading of another instrument, however remote." (Bell 1964, in Bell 2004, p. 20).

The ambiguity in the Bell passage notwithstanding, however, the logic underlying the Boughn claims is not correct. In the opening lines of his 1964 paper, the Bell formulation does not suffer from ambiguities:

The paradox of Einstein, Podolsky and Rosen was advanced as an argument that quantum mechanics could not be a complete theory but should be supplemented by additional variables. These additional variables were to restore causality and locality. In this note that idea will be formulated

\footnotetext{
${ }^{7}$ In the same sense the term Classicality was also adopted by Werner in his critical comment of the Maudlin contribution to the 2014 issue of Journal of Physics A dedicated to 50 years since the Bell theorem (see Maudlin 2014, Werner 2014): this exchange is also illuminating on the issues at stake when discussing the general meaning of Bell's theorem.
} 
mathematically and shown to be incompatible with the statistical predictions of quantum mechanics. It is the requirement of locality, or more precisely that the result of a measurement on one system be unaffected by operations on a distant system with which it has interacted in the past, that creates the essential difficulty. (Bell 1964, in Bell 2004, p. 14).

To make it short ${ }^{8}$, the EPR argument can be formulated as an inference from three conditions to the incompleteness of quantum mechanics: the first is consistency with the statistical predictions of quantum mechanics, the second is the infamous "element-of-physical-reality" condition, whose original formulation in the EPR paper Einstein was dissatisfied with (Howard 1985), and the third is of course locality. It must be stressed that assuming the "element-of-physical-reality" condition is not equivalent to assuming the existence of elements-of-physical-reality (the 'classicality' Boughn seems to refer to) as an autonomous condition: on the contrary, assuming this condition simply amount to require a criterion in order for a property of a physical system to be an objective (i.e. measurement-independent) property 9 . The effective existence of such properties is rather a consequence of the locality assumption. Since we start with an entangled state of a composite system, in which spin properties of each EPR subsystem are not elements of physical reality and we end dealing with post-measurement states in which such properties are indeed elements of physical reality, the only option open to a local description of the whole process is that those properties were already there, and this is something we derive from our assumption that all physical processes involved in the EPR-preparation-and-measurement procedure must be local. But if such existence of elements-of-physical-reality (the Boughn 'classicality') is not assumed at the outset, we cannot dismiss anymore the Bell theorem as a nonlocality result simply by charging it with the accusation of smuggling some 'classical realism' - whatever it is - into the description since the beginning. If this is true, then we must acknowledge that what the Bell theorem is about is nonlocality.

We need no great effort to support this conclusion. We just need to read properly the Bell text that follows immediately the opening lines of his 1964 paper we referred to above, where Bell effectively summarizes the EPR argument:

Consider a pair of spin one-half particles created somehow in the singlet spin state and moving freely in opposite directions. Measurements can be made, say by Stern-Gerlach magnets, on selected components of the spins $\sigma 1$ and $\sigma 2$. If measurement of the component $\sigma 1 \cdot a$, where a is some unit vector, yields the value +1 then, according to quantum mechanics, measurement of $\sigma 2 \cdot$ a must yield the

\footnotetext{
${ }^{8}$ The details can be found in Laudisa 2008, pp. 1118-1122.

${ }^{9}$ Redhead 1987, Ghirardi, Grassi 1994, Norsen 2007, Laudisa 2008, 2012, Maudlin 2014.
} 
value -1 and vice versa. Now we make the hypothesis, and it seems one at least worth considering, that if the two measurements are made at places remote from one another the orientation of one magnet does not influence the result obtained with the other. Since we can predict in advance the result of measuring any chosen component of $\sigma 2$, by previously measuring the same component of $\sigma 1$, it follows that the result of any such measurement must actually be predetermined. Since the initial quantum mechanical wave function does not determine the result of an individual measurement, this predetermination implies the possibility of a more complete specification of the state. (Bell 1964, in Bell 2004, pp. 14-15], italics added)

As should be clear from a fair reading of the Bell original article, the Bell theorem starts exactly from the alternative established by the EPR-Bohm argument-namely, locality and completeness cannot stand together-and goes for the proof that, whatever form the completability of quantum mechanics might assume, the resulting theory cannot preserve the statistical predictions of quantum mechanics and be local at the same time: this means that neither a pre-existing-property assumption (or 'Objectivity' or 'Classicality' or whatever synonymous one likes to choose) nor a determinism assumption are assumed in the derivation of the original Bell inequality.

In addition to the logical status of the premises of the Bell theorem, there is a deep motivation for restricting the attention to locality. In the area of investigations opened nearly half a century ago by John S. Bell, the question naturally arose of what would have been the implications of extending quantum mechanics, in view of the emergence of phenomena that were not easy to accommodate within a familiar view of the physical world, non-locality being the most urgent case. Due to the unavoidable existence of entangled states - something that makes quantum mechanics a non-local theory in a fundamental sense due to the linearity of the theory, of which entanglement is a consequence (more on this later) - it has seemed plausible to put things in the following way: let us ask whether quantum mechanics might be seen as a 'fragment' of a more general theory which - at a 'higher' level - may recover that locality that turns out not to hold at the strictly quantum level. One of the strong points of the original Bell strategy that led to the Bell-named theorem was exactly that this hypothetical extension was confined to the locality/non-locality issue and needed not say anything on further details concerning 'realistic' or 'non-realistic' properties, states or whatever: in addition to being useful for the economy of the theorem, this point was absolutely plausible since it makes sense to require from the extension the only condition that we are interested to add to the new hypothetical super-theory, namely locality. 


\section{3. 'Classicality', counterfactual definiteness and locality}

In addition to the alleged role of 'classical realism' we referred to above, a related claim has been around for decades now. According to this claim, the Bell result ensuing from the EPR argument need not be interpreted in terms of nonlocality since it depends on a counterfactual type of reasoning which would be legitimate only in a 'classical' context; for its supporters, this is another motivation for taking the Bell theorem as a 'no-classicality' rather than a nonlocality result.

Boughn in his paper supports this claim:

Bell's theorem clearly makes use of counterfactual definiteness; his inequality involves the correlations of the spins of the two particles in each of two different directions that correspond to noncommuting spin components. This use of counterfactuals is entirely appropriate because it is used to investigate a test for classical hidden variable theories.

What is the logic in this case? Take an ordinary EPR situation with spin $1 / 2$ particles. According to the condition of reality, the choice of the experimenter on one side to measure spin along the $z$-axis allows her to predict with certainty the outcome of the same measurement on the other side and, as a consequence, to conclude that the outcome of this measurement corresponds to an element of physical reality there. The choice could have been different, though: the $x$-axis could have been selected as the axis along which the experimenter could perform the spin measurement. The conclusion would have been in this case that the element of physical reality on the other side was the outcome of the measurement along the same axis $x$. Since in fact the experimenter can only perform one measurement at a time, she cannot conclude that both the measurement outcomes (along the $z$-axis and along the $x$-axis) correspond to elements of physical reality on the other side, but rather only that one or the other does. To assume instead that both elements of physical reality are fixed even if quantum mechanics prevents that - so the argument concludes - is essentially equivalent to assume again a form of classicality (the first formulation of this argument is probably due to Peres 1978).

The flaw in this argument is similar to the one discussed in the previous section, namely the supposition that the existence of the elements of physical reality is added on top with no other justification than to show that this 'classical-like' supposition cannot hold in a quantum 
world. If, on the other hand, we take seriously (i) the fact that it is the locality assumption that is prior, and (ii) that it is exactly this assumption that legitimates to talk of elements of physical reality corresponding to spin measurements along different axes, we realize that the above appeal to counterfactual reasoning does no harm. If the theory by which we try to account for EPR correlation is assumed to be local, then the choice of a certain axis for spin measurement here is totally unaffacted by what is the chosen axis for spin measurement there, and this holds perfectly the same also in a counterfactual sense. Suppose in the actual world the experimenter has chosen the axis $z$ : if her measuring operations cannot affect by definition what axis is chosen - and what outcome has been obtained - on the other side, this holds naturally for whatever choice and this makes it invalid to claim that in an ordinary EPR argument we have adopted a conterfactual definiteness assumption in addition to locality.

\section{Locality, outcome independence and quantum mechanics}

In a section of his paper Boughn focuses on the so-called principle of separation (Trennungsprinzip), an assumption that Einstein started to use instead of the infamous condition for the elements of physical reality after 1935, unhappy as he was of the formulation of the incompleteness argument for quantum mechanics in the EPR paper (cp. again Howard 1985). The principle (that we will denote by SEPEinst) reads as follows:

After the collision, the real state of (AB) consists precisely of the real state A and the real state of $\mathrm{B}$, which two states have nothing to do with one another. The real state of $B$ thus cannot depend upon the kind of measurement I carry out on A [SEPEinst]. But then for the same state of B there are two (in general arbitrarily many) equally justified $\Psi_{\mathrm{B}}$, which contradicts the hypothesis of a one-to-one or complete description of the real states. (Einstein 1935, cit. in Howard 2007)

In fact, the above passage turns out to be an extra-short version of the post-EPR incompleteness argument defended by Einstein, in which completeness is conceived as a oneto-one correspondence between the wave function and the 'real state' of the system (as a matter of fact, this notion of 'real state' is no less mysterious than the EPR reality condition from which Einstein meant to depart). In this version, quantum mechanics is shown to be incomplete because the possibility of different choices of a measurement on one side licenses 
a plurality of wave functions associated to the 'real state' of tyhe system on the other side, a plurality that violates the above one-to-one correspondence (Howard 2007).

Independently of the fate of the several Einsteinian incompleteness arguments, Boughn proposes an 'experimentalist' reading of the separation principle (let us call it SEPExp), according to which if systems $A$ and $B$ are spatially separated, then a measurement of system A can, in no way, have any effect on any possible measurement of system B (Boughn 2017, p. 6), and claims that, while the principle SEPEinst requires "that a measurement of system A can have no effect on the state of system B", the principle SEPExp requires "that a measurement of system A can have no effect on the result of any measurement on system B", a condition that in Boughn's view - is not violated in standard quantum mechanics (Boughn 2017, p. 6, italics in the original). Some remarks are in order. First, the translation of SEPEinst into SEPExp can hardly be conceived as a gain in conceptual clarity: while the Einsteinian notion of (in)dependence between states can be provided with a definite standard meaning, no matter what Einstein might have meant with the expression 'real state', the same cannot be said of the notion of (in)dependence between measurements, if not within a detailed model of the measurement process that Boughn does not mention. Second, this lack of clarity about the (in)dependence between measurements prevents from really grasping what the requirement "a measurement of system A can have no effect on the result of any measurement on system B" might amount to. The shift from the term measurement to the term result in the above requirement may suggest a comparison of the Boughn argument with a distinction that was introduced long time ago, apparently in order to achieve what was known as a 'peaceful coexistence' between quantum mechanics and relativity theory about nonlocality: the distinction between parameter independence and outcome independence in the context of stochastic hidden variable models of quantum mechanics (Shimony 1984).

Let us employ the symbol $\lambda$ to denote all parameters useful to characterize the complete specification of the state of an individual physical system (the presentation follows Ghirardi et al. 1993). In a standard EPR-Bohm-like situation, the expression

$$
\mathrm{P}_{\lambda}{ }^{\mathrm{LR}}(x, y, \mathrm{n}, \mathrm{m})
$$

denotes the joint probability of getting the outcome $x(x= \pm 1)$ in a measurement of the spin component along $\mathrm{n}$ at the left $(\mathrm{L})$, and $y(y= \pm 1)$ in a measurement of the spin component along $\mathrm{m}$ at the right $(\mathrm{R})$ wing of the apparatus. We assume that the experimenter at $\mathrm{L}$ can make a free-will choice of the direction $\mathrm{n}$ and similarly for the experimenter at $\mathrm{R}$ and the 
direction $\mathrm{m}$. Both experimenters can also choose not to perform the measurement. Bell's locality assumption can be expressed as

$$
\mathrm{P}_{\lambda}{ }^{\mathrm{LR}}(x, y ; \mathrm{n}, \mathrm{m})=\mathrm{P}_{\lambda} \mathrm{L}\left(x, \mathrm{n},{ }^{*}\right) \mathrm{P}_{\lambda}{ }^{\mathrm{R}}\left(y ;{ }^{*}, \mathrm{~m}\right)
$$

where the symbol * in the probabilities at the r.h.s denotes that the corresponding measurement is not performed ${ }^{10}$. Jarrett has shown that condition (1.2) is equivalent to the conjunction of two logically independent conditions, namely (Jarrett 1984)

$$
\mathrm{P}_{\lambda} \mathrm{L}(x, \mathrm{n}, \mathrm{m})=\mathrm{P}_{\lambda} \mathrm{L}\left(x, \mathrm{n},{ }^{*}\right)
$$

$$
\mathrm{P}_{\lambda}{ }^{\mathrm{R}}(y, \mathrm{n}, \mathrm{m})=\mathrm{P}_{\lambda}{ }^{\mathrm{R}}(y ; *, \mathrm{~m})
$$

and

$$
\mathrm{P}_{\lambda}{ }^{\mathrm{LR}}(x, y, \mathbf{n}, \mathrm{m})=\mathrm{P}_{\lambda} \mathrm{L}(x, \mathbf{n}, \mathrm{m}) \mathrm{P}_{\lambda}^{\mathrm{R}}(y, \mathbf{n}, \mathrm{m})
$$

Conditions (1.3a) - referred to as parameter independence (PI) - jointly express the requirement that the probability of getting a result at $L(R)$ is independent from the setting chosen at R (L), while condition (1.3b) - referred to as outcome independence (OI) expresses the requirement that the probability of an outcome at one wing does not depend on the outcome which is obtained at the other wing. Now we assume - a reasonable move, I think - that we make sense of the Boughn requirement "that a measurement of system A can have no effect on the result of any measurement on system B", allegedly presupposed by SEPExp, in terms of OI: under this assumption, however, it can be shown that standard quantum mechanics does violate it. For in the standard EPR case when $\lambda$ is the singlet state $\Psi$, if we choose $\mathbf{n}=\mathbf{m}$ we get

$$
\begin{aligned}
& \mathrm{P}_{\Psi}{ }^{\mathrm{LR}}(1,-1 ; \mathrm{n}, \mathrm{n})=\mathrm{P}_{\Psi}{ }^{\mathrm{LR}}(-1,1 ; \mathrm{n}, \mathrm{n})=1 / 2 \\
& \mathrm{P}_{\Psi}{ }^{\mathrm{LR}}(1,1 ; \mathrm{n}, \mathrm{n})=\mathrm{P}_{\Psi}{ }^{\mathrm{LR}}(-1,-1 ; \mathrm{n}, \mathrm{n})=0
\end{aligned}
$$

but for any $x, y$

$$
\mathrm{P}_{\Psi}^{\mathrm{L}}(x, \mathbf{n}, \mathbf{n}) \mathrm{P}_{\Psi}^{\mathrm{R}}(y, \mathbf{n}, \mathbf{n})=1 / 4
$$

a result that shows the quantum mechanical violation of outcome independence for certain choices of parameters. This allows us to draw two conclusions: first, if we suppose that the distinction between PI and OI captures more precisely the vague principle behind SEPExp, it is not true that standard quantum mechanics does not contemplate violations of such principle.

\footnotetext{
10 In order to make the this intuitive idea more formally precise, we might employ the notion of quantummechanical non-selective measurement. I am grateful to the referee for pressing this clarification.
} 
As a consequence, and this is the second point, standard quantum mechanics already embodies a certain rate of nonlocality anyway, even if for the sake of discussion we put into bracket the more general Bell nonlocality theorem (according to which any theory whatsoever incorporating the statistical predictions of quantum mechanics is bound to be nonlocal) ${ }^{11}$.

\section{Does entanglement holds in classical physics? On an argument by W.H. Furry}

In a further section on the relation between entanglement and nonlocality, Boughn argues that entanglement does not point to a peculiar class of interactions, when he writes that "the phenomenon of entanglement is not restricted to quantum mechanics. Two classical systems that interact with each other before moving off in different directions are also entangled. To the extent that the interaction can be completely characterized, one can predict the correlations of all possible measurements made on the two systems whether space-like separated or not." (Boughn 2017, p. 12). In support of this surprising claim, he employs a highly ambiguous argument of W.H. Furry, contained in a written report of a public debate on the foundations of quantum mechanics which took place at the Xavier University in 1962 and conceived as an illustration of the EPR argument. Let us see the entire Furry 'reworking' of the so-called paradox in terms of a 'classical', macroscopic ordinary situation:

First, you get two envelopes. Then some person, who becomes incommunicado or commits suicide immediately afterwards, takes one or the other of two playing cards, the red or the black, (we don't know which) and tears it in two, and puts half in each envelope. One of the envelopes is sent to Chicago and at any time we can tell what the color of the half card in that envelope in Chicago is just by opening the envelope we have here. We can tell it instantaneously. It doesn't matter if they are opening the envelope in Chicago simultaneously with the one we have here, or before, or after. They will always correlate. This correlation was established in a way that didn't involve any violation of

\footnotetext{
11 Finally, as far as the PI/OI distinction is concerned, we should recall that standard quantum mechanics although violating OI - does satisfy PI (Ghirardi et al. 1980). On the other hand, Bohmian mechanics displays a reverse behaviour with respect to standard quantum mechanics concerning the distinction PI/OI. PI is clearly violated in principle, due to the very construction of the theory, whereas OI is preserved, since in the deterministic framework of Bohmian mechanics the outcomes are fixed and cannot depend on each other. As is well-known, the role of this distinction within standard quantum mechanics provided in the past some ground for the so-called peaceful coexistence thesis, recalled above (Shimony 1984). According to this thesis, quantummechanical non-locality would not be so harmful: the outcomes are somehow non-locally affecting each other and this seems to threaten the prescriptions of special relativity, but such outomes are uncontrollable and thus we cannot exploit them to produce any robust action-at-a-distance. The effectiveness of the PI/OI distinction in carrying the burden of such an ambitious coexistence has been often questioned: see Maudlin 1994, 20113, pp. 85-90, for a critical analysis.
} 
relativity, because they were both together at the time they were put into the envelopes. (C-F-QM 1962, pp. 88-89)

Boris Podolsky, who was also at the debate, rightly replies that "our opening one envelope to determine what the card is in Chicago does not in any way affect the possibilities in Chicago. While in this quantum mechanical experiment, it does, depending on whether we choose to open one envelope or the other" (C-F-QM 1962, p. 89). But Furry reiterates his point:

It's enough to use, say, two envelopes. We enclose them in a slightly infernal box so that the removing of one of these envelopes from the box will promptly result in the complete obliteration of the other one. Now we have two of these boxes, each with two envelopes. The person tears apart a card out of a deck and puts half in each of these two envelopes. For one of them he chooses a card which is either a black suit or a red suit. For the other one he chooses either a low card or a high card. He puts the black or red in the left -hand envelope, the low or high in the right. Then one box is sent to Chicago and the other is kept here. Now you see, there can never be any contradiction if we pull out the black or red and look at it. The other one is destroyed as soon as we pull it out by the infernal arrangement of the box. If we pull out black or red, we now know that if the corresponding envelope is pulled out in Chicago, we know what the answer will be. If the other envelope is pulled out in Chicago, we don't know anything. In any case, however, the sending of the box is perfectly well understood. There is no contradiction with relativity, and the attaining of information from one place or the other is just what it sounds like. The difference, of course, between the classical and the quantum picture is that the quantum mechanical state does not correspond to this because this nice classical picture of the box with two envelopes is the hidden parameter description and the hidden parameter description is denied in quantum mechanics. But this is the only difference between the two things and there is no difference at all about the questions of information and of distance and time. (C-F-QM 1962, p. 90, italics added)

The italicized part is the part that Boughn cites with approval, noting that "what distinguishes quantum entanglement from its classical counterpart is simply the superposition of states and the quantum interference it implies." (Boughn 2017, p. 12). But such part is nothing but an early formulation of the very same misunderstanding about the role of 'classicality' we have been discussing in the previous pages: the circumstance that in the infernal box example the card color is a definite property well before the act of pulling in Chicago whereas in the EPR scenario spin properties are not definite is a difference that makes the two situations totally irreducible to one another. To defend the opposite is essentially equivalent to arguing that in an EPR scenario we assume at the outset that the spin components are pre-existing properties, a claim that we have previously shown to be incompatible with a correct reading of the EPR-Bell analysis. 


\section{Conclusions}

A die-hard tendency in and out of the area of the foundations of quantum mechanics periodically re-emerges, attempting to claim that after all the Bell theorem did not provide a demonstration that the microphysical world, whatever it is, is nonlocal, but simply an elegant rephrasing of a truism, namely that quantum mechanics is not a classical theory. In a lecture held in 1983 even Richard Feynman claimed that Bell's theorem

is not a theorem that anybody thinks is of any particular importance. We who use quantum mechanics have been using it all the time. It is not an important theorem. It is simply a statement of something that we know is true - a mathematical proof of it. (quoted in Whitaker 2016, p. 493).

Along similar lines, Asher Peres and Daniel Terno have argued that

Bell's theorem asserts that it is impossible to mimic quantum theory by introducing a set of objective local 'hidden' variables. It follows that any classical imitation of QM is necessary nonlocal. However Bell's theorem does not imply the existence of any nonlocality in quantum theory itself." (Peres, Terno 2004, p. 104)

whereas in an article devoted to the EPR argument in the so-called relationalinterpretation of quantum mechanics ${ }^{12}$, Smerlak and Rovelli formulate the issue in the following terms:

In the original 1935 article, the EPR argument was conceived as an attack against the description of measurements in Copenhagen quantum theory and a criticism of the idea that Copenhagen quantum mechanics could be a complete description of reality. Locality and a strong form of realism were given for granted by EPR and completeness was argued to be incompatible with quantum-mechanical predictions. With Bell's contribution, which showed that EPR correlations are incompatible with the existence of a hypothetical complete local realist theory, the argument has been mostly reinterpreted as a direct challenge to "local realism". [...] On the other hand, the Kochen-Specker theorem has questioned the very possibility of uncritically ascribing "properties" to a quantum system. From this perspective, the problem of locality moves to the background, replaced by a mounting critique of strongly objective notions of reality. Here we take this conceptual evolution to what appears to us to be its necessary arriving point: the possibility of reading EPR-type experiments as a challenge to Einstein's strong realism, rather than locality . (Smerlak and Rovelli 2007, p. 427).

Henry P. Stapp once called Bell's theorem "the most profound discovery of science"(Stapp 1975, p. 270). might perhaps have been blamed for over-emphasis. Nevertheless, the flaws of

\footnotetext{
${ }^{12}$ See Laudisa, Rovelli 2013 for a review of this interpretation.
} 
those who downplay it concern both the logical structure of the nonlocality theorem and its conceptual content, and the Boughn 2017 paper exemplifies the most recent reformulation of this stance: the present paper is the $n$-th attempt to show why such stance is likely to miss the meaning of a theorem which is a turning point in framing the image of the quantum world and its consequences.

\section{References}

1. Bell, J.S. 1964, "On the Einstein-Podolsky-Rosen paradox", Physics 1, pp. 195-200 (reprinted in Bell 2004, pp. 14-21).

2. Bell, J.S. 2004, Speakable and Unspeakable in Quantum Mechanics, 2nd edn. Cambridge University Press, Cambridge.

3. Boughn S. 2017, "Making Sense of Bell's Theorem and Quantum Nonlocality", Foundations of Physics, pp. 1-18 (available as First online, doi:10.1007/s10701-0170083-6). 
4. C-F-QM 1962, Conference on the Foundations of Quantum Mechanics, Physics Department, Xavier University.

5. Gachechiladze M., Budroni C., Gühne 0. 2016, "Extreme violation of local realism in quantum hypergraph states", Physical Review Letters 116, 070401.

6. Ghirardi G.C., Rimini A., Weber T. 1980, "A general argument against superluminal transmission through the quantum mechanical measurement process", Lettere al Nuovo Cimento 27, pp. 293-298.

7. Ghirardi G.C., Grassi R., Butterfield J., Fleming G.N. 1993, "Parameter dependence and outcome dependence in dynamical models for state vector reduction", Foundations of Physics 23, pp. 341-364.

8. Ghirardi G.C., Grassi R. 1994, “Outcome predictions and property attribution: the EPR argument reconsidered", Studies in History and Philosophy of Modern Physics 25, pp. 397-423.

9. Goldstein S. 2017, "Bohmian Mechanics", in The Stanford Encyclopedia of Philosophy, E. N. Zalta (ed.),<https://plato.stanford.edu/archives/sum2017/entries/qm-bohm/>.

10. Griffiths R.B. 2011, "EPR, Bell and quantum locality", American Journal of Physics 79, pp. 954-965.

11. Howard D. 1985, "Einstein on locality and separability", Studies in History and Philosophy of Science 16, pp. 171-201.

12. Howard D. 2007, "Revisiting the Einstein-Bohr dialogue", Iyyun: Jerusalem Philosophical Quarterly56, 57-90.

13. Jarrett, J. 1984, "On the physical significance of the locality conditions in the Bell arguments", Nous 18, pp. 569-589.

14. Laudisa F. 2008, "Non-Local Realistic Theories and the Scope of the Bell Theorem", Foundations of Physics,38, 2008, pp. 1110-1132.

15. Laudisa F. 2012, “The uninvited guest: 'local realism' and the Bell theorem" in H. De Regt, S. Hartmann, S. Okasha (eds.), EPSA Philosophy of Science: Amsterdam 2009, Springer 2012, pp. 137-149.

16. Laudisa F., Rovelli C. 2013, "Relational Quantum Mechanics", in The Stanford Encyclopedia of Philosophy (Summer 2013 Edition), E.N. Zalta (ed.), <https://plato.stanford.edu/archives/sum2013/entries/qm-relational/>.

17. Maudlin T. 1994, 20113, Quantum Non-Locality and Relativity. The Metaphysical Intimations of Modern Physics, Wiley-Blackwell, Chichester. 
18. Maudlin T. 2011, "How Bell reasoned: a reply to Griffiths", American Journal of Physics 79, pp. 966-970.

19. Maudlin T. 2014, “What Bell Did”, Journal of Physics A: Math. Theor. 47, 424010.

20. Norsen, T. 2007, “Against 'realism'”, Foundations of Physics 37, pp. 311-340.

21. Pawloski M., Brukner C. 2009, “Monogamy of Bell's inequality violations in nonsignaling theories", Physical Review Letters 102: 0030403.

22. Peres A. 1978, "Unperformed measurements have no result", American Journal of Physics 46, pp. 745-747.

23. Peres A., Terno D. 2004, "Quantum Information and Relativity Theory", Reviews of Modern Physics 76, pp. 93-123.

24. Redhead M. 1987, Incompleteness, Nonlocality and Realism, Clarendon Press, Oxford.

25. Shimony, A. 1984, "Controllable and uncontrollable non-locality", in Kamefuchi, S. et al. (eds.) Foundations of Quantum Mechanics in Light of the New Technology, The Physical Society of Japan, Tokyo (reprinted in A. Shimony, Search for a Naturalistic Worldview, vol. II, pp. 130-139, Cambridge University Press, Cambridge 1993).

26. Stapp H.P., "Bell's Theorem and World Process", Nuovo Cimento, 29B, pp. 270-276.

27. Smerlak M., Rovelli C. 2007, "Relational EPR”, Foundations of Physics 37, pp. 427-445.

28. Werner R. 2014, “Comment on 'What Bell Did'”, Journal of Physics A: Math. Theor. 47, 424011.

29. Whitaker A. 2016, "Richard Feynman and Bell's theorem", American Journal of Physics 84, pp. 493-494.

30. Zukovski M., Brukner C. 2014, “Quantum nonlocality - it ain't necessarily so...”, Journal of Physics A: Math. Theor., 47, 424009. 